\title{
Novel use of esophageal overtube and water flushes as a safety indicator and facilitator for the push technique in esophageal food impaction
}

\author{
Paul P Shao, Jasleen K Grewal, Adam M Custer, Felix Leung \\ VA Greater Los Angeles Healthcare System, UCLA David Geffen School of Medicine, USA
}

Received: June 4, 2020

DOI: $10.5430 /$ dcc.v7n $1 \mathrm{p} 8$
Accepted: July 27, 2020

URL: https://doi.org/10.5430/dcc.v7n1p8
Online Published: August 12, 2020

\begin{abstract}
Esophageal food impaction requires urgent endoscopic intervention either by extraction or pushing into the stomach. Due to risks of perforation, controversy exists between when to extract verses when to push the food bolus. There are no clear guidelines on when it is safe to push a food bolus; endoscopists rely on their subjective experiences and judgment. We present a case with a novel use of an esophageal overtube and water flushes to determine the safety of pushing a food bolus and facilitate passage into the stomach.
\end{abstract}

Key Words: Esophageal food impaction, Esophageal foreign body, EGD, Dysphagia, EGD push technique

\section{INTRODUCTION}

Esophageal foreign body is a common emergency encountered by gastroenterologists. It requires timely assessment and often calls for endoscopic intervention to prevent esophageal wall pressure necrosis and perforation. ${ }^{[1]}$ Food bolus impaction is the most common form of esophageal foreign body in adults. ${ }^{[2]}$ Most food boluses pass into the stomach spontaneously. However, 20\% may become lodged in the esophagus or other locations in the gastrointestinal tract and require endoscopic intervention. ${ }^{[3]}$ The majority of adults that present with esophageal food impaction have underlying esophageal pathology such as benign esophageal stenosis caused by Schatzki rings or peptic strictures, webs, esophagitis, extrinsic compression, or motility disorders. Patients usually present acutely with a clear history of ingestion. Common symptoms include a choking sensation, dyspha- gia, odynophagia, chest pressure, and neck or throat pain. In high-grade esophageal obstruction, patients may experience hypersalivation and may have difficulty swallowing liquids, including their own saliva, necessitating emergent endoscopic intervention.

Extraction and pushing are the main endoscopic methods for food bolus removal. Extraction is performed in a piecemeal fashion with a variety of tools, including forceps, snares, baskets, or graspers. While this method is safe, it can take a long time, especially when the food bolus has been impacted for an extended period of time and is less likely to be grasped as a single piece. The push technique applies gentle pressure on the food bolus with the tip of the endoscope to push the food bolus into the stomach. This method is effective but requires experience and sound judgment due to potential risks of esophageal perforation. There are no clear guidelines on

\footnotetext{
*Correspondence: Paul P Shao; Email: paulpshao@gmail.com; Address: VA Greater Los Angeles Healthcare System, UCLA David Geffen School of Medicine, USA.
} 
when it is safe to attempt the push method. ${ }^{[4]}$ We present a case with the novel use of monitoring the rise of water flushes in an esophageal overtube to determine the safety of pushing a food bolus and facilitate passage of the bolus down into the stomach.

\section{CASE REPORT}

A 69-year-old man presented to the Emergency Department 12 hours after ingesting a piece of steak with a sensation of food stuck in his throat. He had a history of left upper lobe lung adenocarcinoma with radiation three years prior with recurrence in the left upper lobe and mediastinal lymph nodes. He denied drooling or coughing, but was unable to drink any liquids.

Vital signs were stable. Complete blood count and basic metabolic panel were unremarkable. Neck x-ray showed mild prevertebral soft tissue swelling and dilatation of the esophagus without definite radiopaque foreign body.

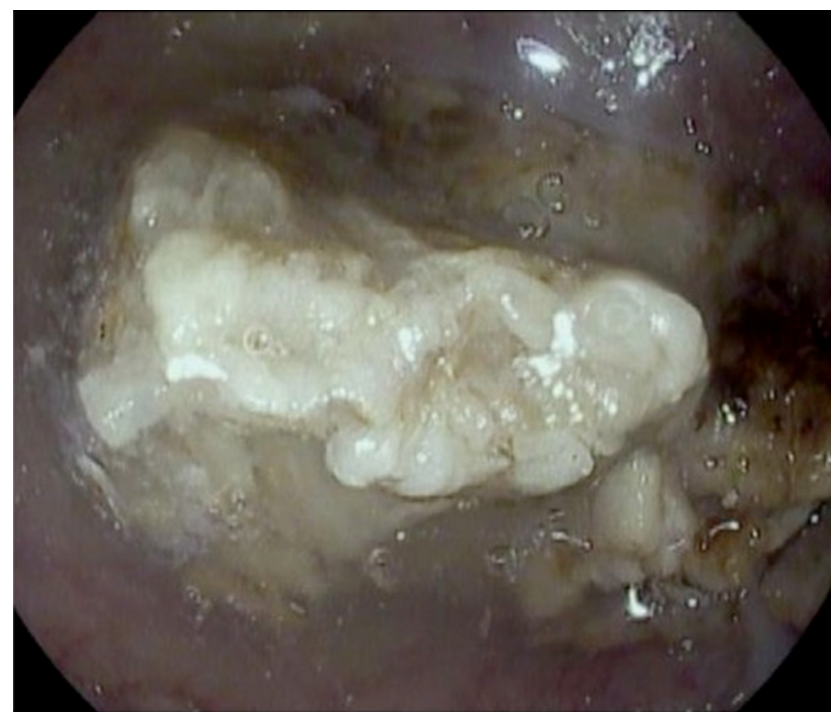

Figure 1. Impacted meat in the esophagus

The patient underwent esophagogastroduodenoscopy (EGD), which showed a large meat bolus firmly fixed in the upper esophagus (see Figure 1). An overtube was placed to protect the airway. Extraction was performed with a combination of large and small rat-toothed forceps, Roth Net and snare in piecemeal fashion. The food bolus was soft and only tiny pieces were retrieved with each attempt. Extraction was attempted for 90 minutes without much progress (see Figure 2). The push method was considered, but the endoscope could not be passed distal to the food bolus. Given the history of radiation and recurrence of lung adenocarcinoma involving the mediastinum, it was uncertain whether it would be safe to push the food bolus. Water flushes were used to help determine when it would be safe to push the bolus. Water was initially retained proximal to the bolus (refluxed back into the overtube), suggesting that there was a complete luminal obstruction and that pushing would be ineffective and unsafe. However, after more of the bolus was extracted (see Figure 3 ), water passed beyond the bolus distally (no longer refluxed back into the overtube), indicating only partial luminal obstruction and that it might be safe to push the food bolus. With a gentle push, the food bolus easily passed into the stomach (see Figure 4). The impaction appeared to be due to a 6-8 $\mathrm{cm}$ area of mild stricturing in proximal esophagus, most likely due to previous radiation. There were no signs of extraluminal compression, and the rest of the mucosa appeared normal.

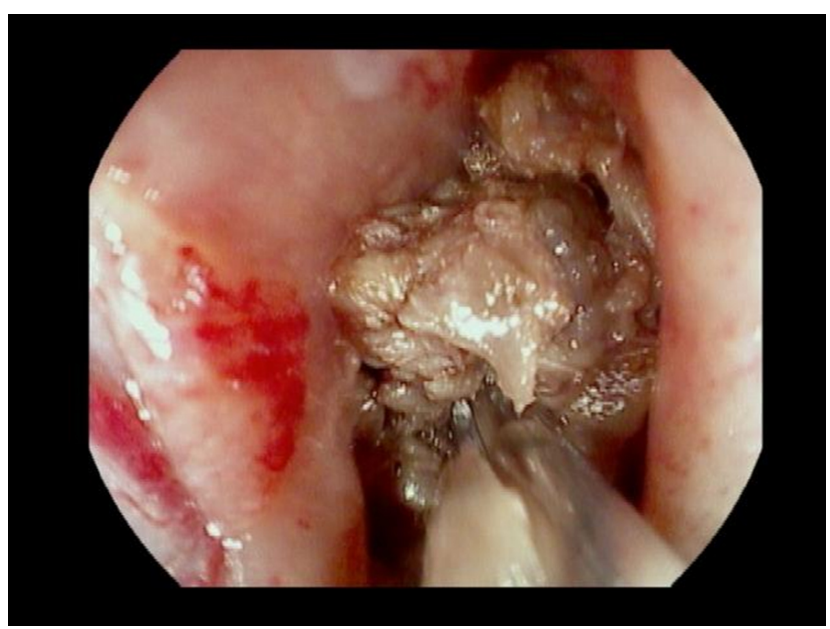

Figure 2. Piecemeal removal of the food bolus with forceps

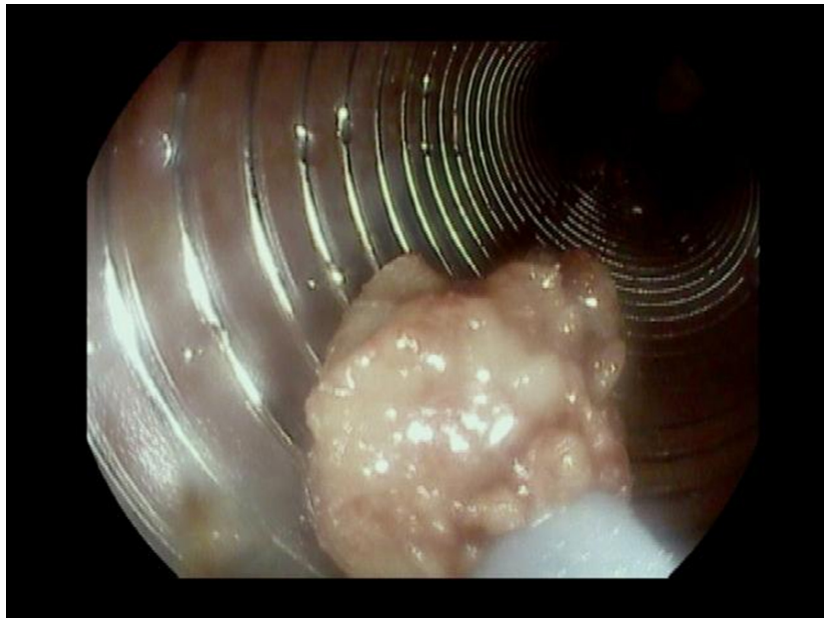

Figure 3. Snare removal of parts of the food bolus 


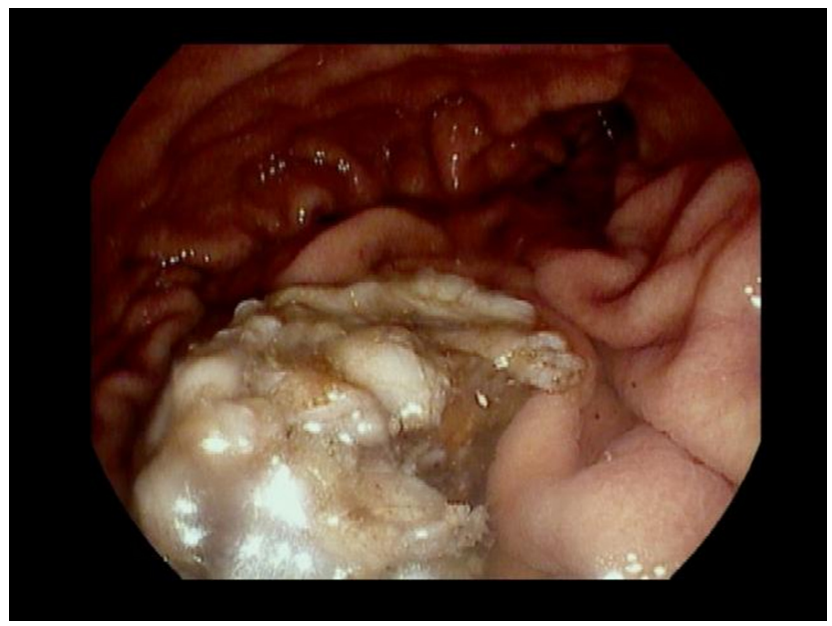

Figure 4. Meat bolus pushed into the stomach

\section{DiscuSSION}

Esophageal food impaction is a common medical emergency. While most foreign body ingestions pass spontaneously, endoscopic intervention is required in $10 \%-20 \%$ of cases. ${ }^{[5]}$ The most common underlying causes include Schatzki rings, esophageal or gastric strictures, esophageal cancer, hiatal hernias, and achalasia. However, approximately 10\%-20\% of patients have no structural abnormality. ${ }^{[6,7]}$ A study of 189 patients with acute esophageal impaction found $41 \%$ had a Schatzki ring, 32\% had an esophageal stricture, 2\% had esophageal cancer, and $25 \%$ had no structural abnormality. ${ }^{[6]}$ Recently, eosinophilic esophagitis has been recognized as a main cause of dysphagia and impaction, especially in men under the age of 50. ${ }^{[-10]}$ In a 2019 study of 214 food impactions at the Mayo Clinic, eosinophilic esophagitis accounted for $18.7 \%$ of the cases and stricture accounted for $24.3 \%$. ${ }^{[11]}$

Esophageal food impaction requires urgent endoscopic disimpaction, typically within 24 hours (Eisen). Food boluses can be removed by extraction in a piecemeal fashion or by gentle manipulation into the stomach. Historically, food extraction is widely advocated and pushing has been avoided due to concerns for associated distal obstructed lesions and increased risks for perforation. However, several studies have demonstrated that push method is both safe and effective. In 62 food impaction cases, Katsinelo et al. found that more than half of the food boluses were successfully removed with either the push method or push-plus-extraction. ${ }^{[12]}$ A retrospective study of 189 patients with acute esophageal food impaction found the push method had a success rate of $97 \%$ without any significant complications, such as perforation, bleeding, or aspiration. ${ }^{[6]}$ In a separate study with 223 episodes of impaction among 194 patients, the push technique was successful alone or in combination with extraction in $84 \%$ of cases. ${ }^{[13]}$ Another study reported a $100 \%$ success rate with the push method in 48 adults with food impaction without any complications. ${ }^{[7]}$ The Mayo Clinic also reported no difference in adverse events between extraction and the push technique. ${ }^{[11]}$

The push technique is recommended as the primary method to treat food bolus impaction and is advocated in patients without a history of dysphagia or underlying structural abnormality. ${ }^{[5]}$ It is especially useful when the food bolus is soft and easily broken into fragments, which makes extraction difficult. $^{[14]}$ To ensure the safety of the push technique and prevent perforation, the endoscope should be inserted beyond the bolus to demonstrate the absence of distal obstruction or stricture. ${ }^{[5,15]}$ A modified push technique has been described where a Savary-Gilliard dilator is inserted via a guide wire past the food bolus to encourage passage. ${ }^{[1,7]}$ However, both rely on the ability of the endoscope to steer beyond the bolus. No clear guidelines exist on when it is safe to attempt the push method when the endoscope is unable to pass distal to the bolus. Endoscopists rely on their subjective experiences and best judgments. This case demonstrates a novel use of esophageal overtube and water flushes to assess when it is safe to push the food bolus. If water is retained and refluxed into the overtube, there may be a complete luminal obstruction and pushing is not recommended. On the other hand, if water does not accumulate or reflux into the overtube, there is an opening in the lumen allowing the water travel distal to the bolus and pushing the bolus may be considered. Moreover, water may moisturize the food bolus and help loosen the impaction to facilitate the bolus down the stomach. It is important to note that water flushes may increase the risk of aspiration. However, utilization of an overtube protects the airway, minimizes aspiration risk, and allows for multiple passes of the endoscope during bolus retrieval. ${ }^{[5]}$

The use of an esophageal overtube and water flushes provides an additional safety indicator to help decide when to push the food bolus. While a single case study may not be generalizable, especially in patients with underlying structural abnormality, the use of an esophageal overtube and water flushes is a safe, easy and cost-effective method to complement the push technique that endoscopists may utilize in the management of food impaction.

\section{CONFlicts of InTEREST Disclosure}

The authors declare no conflicts of interest. 


\section{REFERENCES}

[1] Eisen GM, Baron TH, Dominitz JA, et al. Guideline for the management of ingested foreign bodies. Gastrointest Endosc. 2002; 55: 802-6. https://doi.org/10.1016/S0016-5107 (02) 70407-0

[2] Bekkerman M, Sachdev AH, Andrade J, et al. Endoscopic Management of Foreign Bodies in the Gastrointestinal Tract: A Review of the Literature. Gastroenterol Res Pract. 2016; 2016: 8520767. PMid:27807447. https ://doi .org/10.1155/2016/8520767

[3] Nandi P, Ong GB. Foreign body in the oesophagus: review of 2394 cases. British Journal of Surgery. 1978; 65(1): 5-9. PMid:623968. https://doi.org/10.1002/bjs. 1800650103

[4] Ko HH, Enns R. Review of food bolus management. Can J Gastroenterol. 2008; 22(10): 805-8. PMid:18925301. https : //doi .org/10 $.1155 / 2008 / 682082$

[5] Birk M, Bauerfeind P, Deprez PH, et al. Removal of foreign bodies in the upper gastrointestinal tract in adults: European Society of Gastrointestinal Endoscopy (ESGE) Clinical Guideline. Endoscopy. 2016; 48(5): 489-496. PMid:26862844. https://doi.org/10.1 055/s-0042-100456

[6] Joseph JV, John FJ, James TF. Outcomes of acute esophageal food impaction: success of the push technique. Gastrointestinal Endoscopy. 2001; 53(2): 178-181. PMid:11174288. https ://doi .org/10.1 $067 /$ mge. 2001.111039

[7] Weinstock LB, Shatz BA, Thyssen SE. Esophageal food bolus obstruction: evaluation of extraction and modified push techniques in 75 cases. Endoscopy. 1999; 31(6): 421-5. PMid:10494678. https : //doi.org/10.1055/s-1999-39

[8] Straumann A, Bussmann C, Zuber M, et al. Eosinophilic esophagitis: analysis of food impaction and perforation in 251 adolescent and adult patients. Clinical Gastroenterology and Hepatology. 2008; 6(5): 598-600. PMid:18407800. https://doi.org/10.1016/j. cgh. 2008.02.003
[9] Byrne KR, Panagiotakis PH, Hilden K, et al. Retrospective analysis of esophageal food impaction: differences in etiology by age and gender. Digestive Diseases and Sciences. 2007; 52(3): $717-$ 721. PMid:17253139. https ://doi.org/10.1007/s10620-006 $-9499-0$

[10] Kapel RC, Miller JK, Torres C, et al. Eosinophilic esophagitis: a prevalent disease in the United States that affects all age groups. Gastroenterology. 2008; 134(5): 1316-1321. PMid:18471509. https : //doi.org/10.1053/j.gastro.2008.02.016

[11] Melendez-Rosado J, Corral JE, Patel S, et al. Esophageal Food Impaction. Journal of Clinical Gastroenterology. 2019; 53(3): 179-183. PMid:29517706. https://doi.org/10.1097/MCG.0000000000 001004

[12] Katsinelos P, Kountouras J, Paroutoglou G, et al. Endoscopic techniques and management of foreign body ingestion and food bolus impaction in the upper gastrointestinal tract: a retrospective analysis of 139 cases. J Clin Gastroenterol. 2006; 40: 784-789. PMid:17016132. https://doi.org/10.1097/01.mcg.0000225602.25858.2c

[13] Longstreth GF, Longstreth KJ, Yao JF. Esophageal food impaction: Epidemiology and therapy. A retrospective, observational study. Gastrointest Endosc. 2001; 53: 193-8. PMid:11174291. https : //doi.org/10.1067/mge.2001.112709

[14] Webb WA. Management of foreign bodies of the upper gastrointestinal tract. Gastroenterology. 1988; 94(1): 204-216. https : //doi.org/10.1016/0016-5085(88)90632-4

[15] Fung BM, Sweetser S, Kee Song LMW, et al. Foreign object ingestion and esophageal food impaction: An update and review on endoscopic management. World Journal of Gastrointestinal Endoscopy. 2019; 11(3): 174. PMid:30918584. https://doi.org/10.4253/wjge .v11.i3.174 